\title{
Zero-point fluctuations in the ground state of a mesoscopic normal ring
}

\author{
Pascal Cedraschi and Markus Büttiker \\ Département de Physique Théorique, Université de Genève, \\ 24, quai Ernest Ansermet, CH-1211 Geneva 4, Switzerland
}

(October 26, 2018)

\begin{abstract}
We investigate the persistent current of a ring with an in-line quantum dot capacitively coupled to an external circuit. Of special interest is the magnitude of the persistent current as a function of the external impedance in the zero temperature limit when the only fluctuations in the external circuit are zero-point fluctuations. These are time-dependent fluctuations which polarize the ringdot structure and we discuss in detail the contribution of displacement currents to the persistent current. We have earlier discussed an exact solution for the persistent current and its fluctuations based on a Bethe ansatz. In this work, we emphasize a physically more intuitive approach using a Langevin description of the external circuit. This approach is limited to weak coupling between the ring and the external circuit. We show that the zero temperature persistent current obtained in this approach is consistent with the persistent current calculated from a Bethe ansatz solution. In the absence of coupling our system is a two level system consisting of the ground state and the first excited state. In the presence of coupling we investigate the projection of the actual state on the ground state and the first exited state of the decoupled ring. With each of these projections we can associate a phase diffusion time. In the zero temperature limit we find that the phase diffusion time of the excited state projection saturates, whereas the phase diffusion time of the ground state projection diverges.
\end{abstract}

PACS numbers: 73.23.Ra, 73.23.Hk, 71.27.+a

\section{INTRODUCTION}

An interesting aspect of quantum systems is the fact that even at zero temperature there are fluctuations which manifest themselves in observables which do not commute with the Hamiltonian of the system. In mesoscopic systems phase coherence plays an essential role, and it is thus important to ask to what extent coherence is affected by zero-point fluctuations. To investigate this question we consider the ground state of a normal mesoscopic ring threaded by an Aharonov-Bohm flux and capacitively coupled to an external circuit. Such a ring exhibits a persistent current which is a direct consequence of phase coherent electron motion over distances large compared to the ring circumference. At zero temperature such a ring interacts with an external circuit (see Fig. 1) only due to zero-point fluctuations. More precisely, the external circuit can, through the generation of zero-point voltage fluctuations, induce polarization fluctuations in the ring which in turn affect the magnitude of the persistent current. The source of the voltage fluctuations in the external circuit are the resistive elements. We can thus ask: How does the persistent current of the ring depend on the resistive properties of the external circuit? For the system shown in Fig. 1, Ref. 11 provided an answer by mapping a simple model of a ring with a quantum dot and external circuit on the anisotropic Kondo model and using the known Bethe ansatz solutions of this problem. 2 The purpose of this work is to consider the same model and to provide a discussion which is physically more transparent. The discussion given below is, however, limited to the case of weak coupling between the ring and the external circuit.

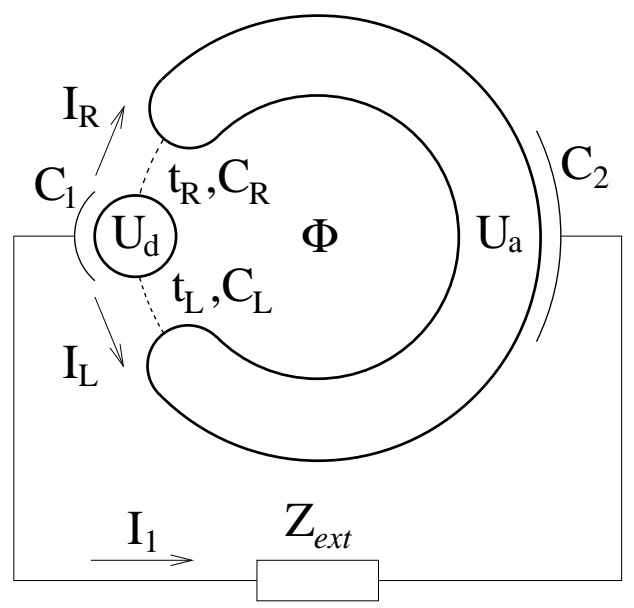

FIG. 1. Ring with an in-line dot subject to a flux $\Phi$ and capacitively coupled to an external impedance $Z_{\text {ext }}$.

Investigations of the coherence properties of the ground state of mesoscopic structures are rare and foncern mainly superconductors. Hekking and Glazman ${ }^{3}$ investigate a thin superconducting loop in an electromagnetic environment; Oshikawa and Zagoskin 1 consider a superconducting grain coupled to a normal wire. In contrast, investigations on dephasing in systems driven out of the ground state are numerous and there exists a considerable literature. In particular, zero-point fluctuations have been of interest, following recent experimental work by Mohanty, Jariwala, and Webb目 on weak localization in metallic diffusive wires. These experiments probe a 
transport state and not the ground state of the system. In the experiments of Mohanty and Webb it is the weaklocalization effect in the linear response conductance of the system which is of interest. Subsequently to these experiments the role of zero-point fluctuations in weak localization effects has been very much debated 69 and a number of works have appeared which suggest that the experiments can possibly be better explained without invoking zero-point fluctuations. 10 Weak-localization is a quantum effect which survives ensemble averaging and thus the dephasing rates which count are specific to the fact that we deal with time-reversed trajectories and that an ensemble average has to be performed. The absence of an effect of zero-point fluctuations found in Refs. [7, 8 might give rise to the mistaken impression that zero-point fluctuations are quite generally without any effect on the coherence properties of a system. The example presented here shows that zero-point fluctuations are clearly important for the coherence properties of the system, although perhaps not for weak localization phenomena. Below we consider a specific ring (not an ensemble) and ask how the maximum amplitude of the persistent current is affected by zero-point fluctuations.

The effect of a thermal bath on the persistent current has been discussed by Landauer and Büttiker 1 and Büttiker12, 13 within a Debye relaxation approach. In this approach, however, the bath affects essentially only the population of different states, but not the electronic states of the ring itself. As a consequence, at zero temperature, this model exhibits no effect due to zero point fluctuations: the magnitude of the persistent current is independent of the coupling strength to the bath. Still a different model, introduced by Büttiker,14 considers a ring coupled via a side branch to a normal electron reservoir. Due to the connection between reservoir and ring a carrier in the ring eventually escapes to the reservoir and is replaced with an incident carrier with a phase which is unrelated to that of the escaping carrier. This model predicts even at zero temperature an amplitude of the persistent current which depends on the coupling strength.14 18 This effect is not due to zero-point fluctuations but results from the exchange of carriers between the reservoir and the ring. If the ring is coupled to a side branch of finite length, the side branch can nevertheless generate effects which are similar to that of a reservoir, especially if only ensemble averaged quantities are considered. This is correct only if the side branch has a charging energy which is weak compared to the level spacing.19 If the charging energy is large compared to the level spacing the side branch has no effect on the ensemble averaged persistent current.19 It is thus interesting to ask, whether there exist models which are strictly canonical (without carrier exchange with a reservoir) and for which the sample specific persistent current depends nevertheless on the properties of the bath. The model investigated in this work examines a ring which is coupled to the bath only via the long range Coulomb force.

The mesoscopic ring which we consider (see Fig. 1) is divided into two regions by tunneling barriers. It is a ring with an in-line quantum dot.2026. This model allows a simple characterization of the electrostatic potential in terms of only two variables $U_{d}$ (for the dot) and $U_{a}$ (for the arm), and by the charges $Q_{d}$ and $Q_{a}$. The two regions, the dot and the arm of the ring, are coupled via capacitors $C_{1}$ and $C_{2}$ to the external circuit. The external circuit is described by its impedance $Z_{\text {ext }}$. The potential at the capacitor $C_{1}$ is denoted by $V_{0}$, the charge by $Q_{0}$. Likewise, we write the potential and the charge on the capacitor $C_{2}$ as $V_{\infty}$ and $Q_{\infty}$.

\section{LANGEVIN EQUATION}

To be specific, we consider a purely resistive external impedance, $Z_{\text {ext }}=R$. The fluctuations generated by this resistor can be represented as a noise source in parallel with the resistor as shown in Fig. 2. The current $\delta V / R$ through the resistor must be equal to the sum of the current of the noise source $\delta I(t)$ and of the current through the ring-dot structure. In terms of the potential difference $\delta V \equiv V_{0}-V_{\infty}$ across the ring-dot structure we find that the coupling between the ring-dot structure and the external circuit is described by

$$
\delta V / R=-C_{0} \delta \dot{V}+\frac{C_{0}}{C_{i}} \dot{Q}_{d}+\delta I(t) .
$$

Here we introduce the capacitance $C_{0}^{-1} \equiv C_{i}^{-1}+C_{e}^{-1}$ which is the series capacitance of the internal capacitance $C_{i} \equiv C_{L}+C_{R}$ and the external capacitance $C_{e}^{-1} \equiv C_{1}^{-1}+C_{2}^{-1}$, and the charge on the $\operatorname{dot} Q_{d}$. A detailed consideration of the circuit equations leading to Eq. (11) is given in Appendix A. The noise source in parallel to the resistor generates a current noise spectrum, $\left\langle\delta I(\omega) \delta I\left(\omega^{\prime}\right)\right\rangle \equiv 2 \pi \delta\left(\omega+\omega^{\prime}\right) S_{I I}(\omega)$, given by

$$
S_{I I}(\omega)=\frac{\hbar \omega}{R} \operatorname{coth}\left(\frac{\hbar \omega}{2 k T}\right),
$$

where $k$ is the Boltzmann constant, and $T$ is the temperature. To complete the description of this system, we need to investigate the dynamics of the ring in the presence of the fluctuating external voltage $\delta V(t)$.

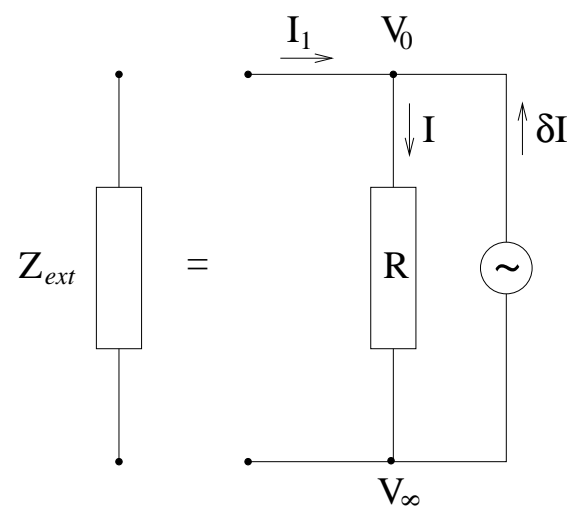


FIG. 2. The external impedance $Z_{\text {ext }}(\omega)$ has been replaced by a resistor $R$ with a noise source in parallel in order to take into account quantum fluctuations.

First, we consider the effect of the external circuit in linear response. Since we are only interested in the weak coupling limit, we can choose the ground state of the ring-dot system in the absence of an external circuit as a state around which we can expand. What we need is the linear response relation between $\delta Q_{d}(t)$ (the deviation of the charge away from a reference state) as a function of the external voltage $\delta V(t)$. Note that both of these variables are in fact operators. Here, we proceed as in the random phase approximation and use a linear response function to describe the connection between these two quantities. In linear response we can Fourier transform $\delta Q_{d}(t)$ and $\delta V(t)$. The linear response coefficient gives the increment of the charge on the dot in response to a variation of an external voltage: It is thus a dynamic capacitance which we denote by $C_{d}(\omega)=\delta Q_{d}(\omega) / \delta V(\omega)$. Similarly, the relation between the charge $\delta Q_{0}$ piled up on the external capacitor $C_{1}$ and the applied voltage is determined by the overall capacitance of the ring dotstructure vis-a-vis the external circuit, and is denoted by $C_{\mu}(\omega)=\delta Q_{0}(\omega) / \delta V(\omega)$. From circuit theory (see Appendix A it follows that these two capacitances are related

$$
C_{\mu}(\omega)=C_{0}-\frac{C_{0}}{C_{i}} C_{d}(\omega)
$$

The noise source (see Fig. 2) sees a total impedance $Z(\omega)$ which consists of this capacitance and the resistance

$$
\frac{1}{Z(\omega)}=\left[\frac{1}{R}-i \omega C_{\mu}(\omega)\right] .
$$

With these response coefficients Eq. (1) leads to

$$
\delta V(\omega)=Z(\omega) \delta I(\omega)=\left[1-i \omega R C_{\mu}(\omega)\right]^{-1} R \delta I(\omega)
$$

and the spectrum of the external voltage is thus

$$
S_{V V}(\omega)=\frac{R \hbar \omega \operatorname{coth}\left(\frac{\hbar \omega}{2 k T}\right)}{\left[1+\omega^{2}\left(R C_{\mu}(\omega)\right)^{2}\right]}
$$

Our next task is now to find explicit expressions for the capacitances $C_{\mu}(\omega)$ and $C_{d}(\omega)$.

\section{A. The dynamics of the ring}

The dynamics of the charge on the dot, determined by $\hat{Q}_{d}$, is in the Heisenberg picture given by

$$
\frac{\partial}{\partial t} \hat{Q}_{d}=\frac{\hbar}{i}\left[\hat{H}_{\text {ring }}, \hat{Q}_{d}\right]
$$

where $\hat{H}_{\text {ring }}$ is the Hamiltonian of the ring in an applied external potential $\delta V$.
We assume that the tunneling amplitudes are much smaller in magnitude than the level spacing in the dot and the level spacing in the arm. Moreover, we assume the electrons to be spinless. As discussed in Refs. 20,21 a spin singlet appears in the case of electrons with spin, and the tunneling amplitude is enhanced by a factor of $\sqrt{2}$ Our choice of parameters excludes the Kondo effect, 24 also if the electron spin is taken into account. As a matter of fact, the Kondo effect appears only at a tunneling amplitudecomparable to or larger than the mean level spacing.25 We note that the number of charge carriers in the ring is conserved. Following Büttiker and Stafford 20,21 we consider hybridization between the topmost occupied electron level in the arm and the lowest unoccupied electron level in the dot, $\epsilon_{a M}$ and $\epsilon_{d(N+1)}$ only. To simplify the notation, we denote the tunneling amplitudes between the levels $\epsilon_{a M}$ and $\epsilon_{d(N+1)}$ by $t_{L}$ for tunneling through the left junction and by $t_{R}$ for tunneling through the right one, and introduce the total tunneling amplitude

$$
\frac{\hbar \Delta_{0}}{2}=\sqrt{t_{L}^{2}+t_{R}^{2} \pm 2 t_{L} t_{R} \cos \frac{2 \pi \Phi}{\Phi_{0}}},
$$

where $\Phi_{0}=h c / e$ is the flux quantum, and the sign in front of the cosine depends on the parity of the number of electrons in the ring. The sign is positive, if the number of electrons is odd, and negative if the number is even. In the model introduced above, we are considering only two states, namely the topmost electron being in the dot, which we represent by the vector $(1,0)$, and the topmost electron being in the arm of the ring, written as $(0,1)$. The dynamics of these two states is described by a time dependent Hamiltonian

$$
\hat{H}_{\text {ring }}=\frac{\hbar \varepsilon(t)}{2} \sigma_{z}-\frac{\hbar \Delta_{0}}{2} \sigma_{x}+\frac{\hbar \nu(t)}{2} \mathbf{1},
$$

where $\sigma_{z}$ and $\sigma_{x}$ are Pauli matrices, and $\mathbf{1}$ is the identity matrix. The prefactor $\hbar \nu(t)=-C_{0} \delta V^{2}(t)$ is a global shift in energy. We split up the detuning $\varepsilon(t)$ into a time independent and a time dependent part $\varepsilon(t)=\varepsilon_{0}+\delta \varepsilon(t)$, with

$$
\begin{aligned}
\hbar \varepsilon_{0} & \equiv \epsilon_{d(N+1)}-\epsilon_{a M}+\frac{e^{2}\left(N-N_{+}+1 / 2\right)}{C}-\frac{C_{e}}{C} V_{0} \\
& \equiv \frac{e}{C}\left(Q_{d *}-Q_{d 0}\right) \\
\delta \varepsilon(t) & \equiv \frac{e}{\hbar} \frac{C_{0}}{C_{i}} \delta V(t) .
\end{aligned}
$$

The effective background charge on the dot is $Q_{d 0}=$ $e N_{+}+C_{e} V_{0}$, where the first term is a built in background charge and the second term can be externally controlled by applying a static voltage across the ring-dot structure. If coherent tunneling is neglected the ground states of the ring with $N$ and $N+1$ carriers are degenerate if the polarization charge is equal to 


$$
Q_{d *} \equiv e\left(N+\frac{1}{2}\right)+\frac{C}{e}\left(\epsilon_{d(N+1)}-\epsilon_{a M}\right) .
$$

Thus $Q_{d 0}=Q_{d *}$ is the (classical) condition at which the Coulomb blockade is lifted. Quantum mechanically the state in the ring and the state in the arm of the ring are in resonance when $Q_{d 0}=Q_{d *}$ and the persistent current exhibits a peak. Our simple two-level picture is applicable when $\varepsilon_{0}$ is small, that is, in the vicinity of a resonance.

We want to find the time evolution of a state $\psi(t)$, which we write as

$$
\psi=e^{i \chi / 2}\left(\begin{array}{l}
\cos \frac{\theta}{2} e^{i \varphi / 2} \\
\sin \frac{\theta}{2} e^{-i \varphi / 2}
\end{array}\right),
$$

with $\theta, \varphi$ and $\chi$ real. This is the most general form of a normalized complex vector in two dimensions. In terms of $\theta, \varphi$ and the global phase $\chi$, the time dependent Schrödinger equation reads

$$
\begin{aligned}
\dot{\varphi} & =-\varepsilon_{0}-\delta \varepsilon(t)-\Delta_{0} \cot \theta \cos \varphi, \\
\dot{\theta} & =-\Delta_{0} \sin \varphi, \\
\dot{\chi} & =-\nu+\Delta_{0} \frac{\cos \varphi}{\sin \theta} .
\end{aligned}
$$

Note that Eq. (16) describing the dynamics of the global phase $\chi$ is special in the sense that it is driven by $\phi$ and $\theta$ but it has no back-effect onto them. Moreover, $\chi$ is irrelevant for expectation values, like the persistent current or the charge on the dot. It plays an important role, however, in the discussion of phase diffusion times, see Sec. IV. The system of the two equations for $\varphi$ and $\theta$, Eqs. (14, 15), is closed by Eq. (11),

$$
\dot{Q}_{d}=\frac{d}{d t}\left\langle\psi(t)\left|\hat{Q}_{d}\right| \psi(t)\right\rangle,
$$

with $\psi(t)$ defined above, and

$$
\hat{Q}_{d}=e\left(\begin{array}{ll}
1 & 0 \\
0 & 0
\end{array}\right)+e N_{+},
$$

We find

$$
\dot{Q}_{d}=e \frac{d}{d t}\left\langle\psi(t)\left|\left(\begin{array}{ll}
1 & 0 \\
0 & 0
\end{array}\right)\right| \psi(t)\right\rangle=-\frac{e}{2} \sin \theta \dot{\theta} .
$$

Using this result and Eq. (11), gives

$$
\delta V=-C_{0} R \delta \dot{V}-\frac{C_{0}}{C_{i}} \frac{e}{2} \sin \theta \dot{\theta}+R \delta I(t) .
$$

Eqs. (14, 15) and (20) form a closed system of equations in which the external circuit is incorporated in terms of a fluctuating current $\delta I(t)$ and of an ohmic resistor $R$. In the next section, we investigate Eqs. (14, 15) and (20) to find the effect of zero-point fluctuations on the persistent current of the ring.

\section{B. Expansion around a stationary state}

First, let us discuss the stationary states of the system of differential equations, Eqs. (14, 15) and (20) in the absence of the noise term $\delta I(t)$. We take $0 \leq \varphi<2 \pi$ and $0 \leq \theta<\pi$. This gives immediately $\sin \varphi=0$ and consequently a stationary state has $\varphi \equiv \varphi_{0}$, with $\varphi_{0}=0$ or $\varphi_{0}=\pi$. With this it is easy to show that in the stationary state we must have $\theta \equiv \theta_{0}$, with

$$
\cot \theta_{0}= \pm \frac{\varepsilon_{0}}{\Delta_{0}} .
$$

The lower sign applies for $\varphi_{0}=0$. This is the ground state for the ring-dot system at fixed $\varepsilon(t) \equiv \varepsilon_{0}$, and the upper sign holds for $\varphi_{0}=\pi$. The energy of the ground state is $-\hbar \Omega_{0} / 2$, thus the global phase is $\chi_{0}(t)=\Omega_{0} t$. Here

$$
\Omega_{0}^{2} \equiv \varepsilon_{0}^{2}+\Delta_{0}^{2}
$$

is the resonance frequency of the (decoupled) two-level system. We also introduce the "classical" relaxation time $\tau_{R C} \equiv R C_{0}$, and a relaxation rate

$$
\Gamma \equiv \pi \alpha \frac{\Delta_{0}^{2}}{\Omega_{0}}
$$

which as we shall see in Sec. IIE is a relaxation rate due to the coupling of the ring-dot system to the external circuit. Here $\alpha$ is a dimensionless coupling constant

$$
\alpha \equiv \frac{R}{R_{K}}\left(\frac{C_{0}}{C_{i}}\right)^{2}
$$

with $R_{K} \equiv h / e^{2}$ the quantum of resistance. We will immediately see the usefulness of these definitions.

Now, we switch on the noise $\delta I(t)$. We seek $\varphi(t), \theta(t)$, $\chi(t)$ and $\delta V(t)$ in linear order in the noise current $\delta I(t)$. We expand $\varphi(t)$ and $\theta(t)$ to first order around the ground state, $\varphi=0$ and $\theta=\theta_{0}$. For $\delta \varphi(t)=\varphi(t)-\varphi_{0}$, $\delta \theta(t)=\theta(t)-\theta_{0}$, we find in Fourier space,

$$
\begin{aligned}
& -i \omega \delta \varphi=-\delta \varepsilon+\frac{\Omega_{0}^{2}}{\Delta_{0}} \delta \theta, \\
& -i \omega \delta \theta=-\Delta_{0} \delta \varphi \\
& -i \omega \delta \varepsilon=\frac{1}{\tau_{R C}}\left[-\delta \varepsilon-\Gamma \delta \varphi+\frac{e}{\hbar} R \frac{C_{0}}{C_{i}} \delta I\right] .
\end{aligned}
$$

We also expand the global phase $\chi(t)$ around its evolution in the ground state $\chi_{0}(t)=\Omega_{0} t$, and define $\delta \chi(t)=\chi(t)-\chi_{0}(t)$. In Fourier space, Eq. (16) becomes

$$
-i \omega \delta \chi=\Omega_{0} \frac{\varepsilon_{0}}{\Delta_{0}} \delta \theta .
$$

We note that there is no effect of the global shift in energy, $\hbar \nu(t)$, as it is quadratic in the voltage $\delta V$, and we are only interested in effects up to linear order in $\delta V$.

In the following section, we evaluate the linear response of the ring, described by Eqs. (25, 26) to an applied external potential $\delta V(\omega)$, giving the frequency dependent capacitance $C_{\mu}(\omega)$. 


\section{Capacitance of the ring and impedance}

We evaluate $\delta Q_{d}(\omega)$ to first order in $\delta V(\omega)$, using Eqs. (25, 26), which gives the dynamic capacitance $C_{d}(\omega)=\delta Q_{d}(\omega) / \delta V(\omega)$,

$$
C_{d}(\omega)=C_{0} \frac{e^{2} /\left(2 C_{i}\right)}{\hbar \Omega_{0}} \frac{\Delta_{0}^{2}}{\omega^{2}-\Omega_{0}^{2}} .
$$

The frequency dependent capacitance as seen from the external circuit reads, see Eq. (3),

$$
C_{\mu}(\omega)=C_{0}\left(1-\frac{e^{2} /\left(2 C_{i}\right)}{\hbar \Omega_{0}} \frac{\Delta_{0}^{2}}{\omega^{2}-\Omega_{0}^{2}}\right) .
$$

The frequency dependent capacitance $C_{\mu}$ contains in addition to the geometrical capacitance $C_{0}$ a term which arises from the dynamic polarizability of the ring. In the low frequency regime the polarizability enhances the capacitance,

$$
C_{\mu}(0)=C_{0}\left(1+\frac{e^{2} /\left(2 C_{i}\right)}{\hbar \Omega_{0}} \frac{\Delta_{0}^{2}}{\Omega_{0}^{2}}\right)=C_{0}+\left(\frac{C_{0}}{C_{i}}\right)^{2} \frac{2 e^{2}\left|t_{ \pm}\right|^{2}}{\left\{\left[e\left(Q_{d 0}-Q_{d *}\right) / C\right]^{2}+4\left|t_{ \pm}\right|^{2}\right\}^{3 / 2}}
$$

The zero frequency capacitance has already been obtained by Stafford and one of the authors.20121 The zerofrequency (electro-chemical) capacitance Eq. (31) can be obtained as asecond order derivative of the grand canonical potential21 with respect to the voltage applied across the ring dot-system or as a dynamic response to a slowly varying voltage across the ring-dot system. Since the tunneling amplitude $\Delta_{0}$ depends periodically on the magnetic flux $\Phi$, see Eq. (8), $C_{\mu}(\omega)$ can be modulated by varying $\Phi$. The electro-chemical capacitance exhibits a peak as the bias $V_{0}$ is varied. The peak shows up at resonance, $Q_{d 0}=Q_{d *}$, corresponding to $\varepsilon_{0}=0$. At the same point, there is also a peak in the persistent current. A recent experiment by Deblock et al.27 demonstrated indeed a flux dependent polarizability of mesoscopic rings.

At high frequencies the polarization cannot follow the external voltage and the capacitance Eq. (30) is smaller than the geometrical capacitance. At $\omega=\Omega_{0}$ the capacitance exhibits a resonance characteristic for dielectric functions. The ring by itself has no damping mechanism, therefore the resonance shows up as a pole. The damping is provided by the dissipation in the external circuit.

From Eqs. (4) and (30), we find

$$
\frac{1}{Z(\omega)}=\frac{1}{R} \frac{\omega^{2}-\Omega_{0}^{2}-i \omega \tau_{R C}\left(\omega^{2}-\Omega_{0}^{2}-\Gamma / \tau_{R C}\right)}{\omega^{2}-\Omega_{0}^{2}} .
$$

This expression for the impedance seen by the noise source contains all the information needed to calculate the various spectral densities.

\section{Spectral densities}

We solve Eqs. (25)-(27) for $\delta \varphi$ by eliminating $\delta \theta$ and $\delta \varepsilon$, and obtain after some algebra

$$
\delta \varphi=\frac{e}{\hbar} \frac{C_{0}}{C_{i}} \frac{(-i \omega) Z(\omega)}{\omega^{2}-\Omega_{0}^{2}} \delta I .
$$

It follows immediately that $\langle\delta \varphi(\omega)\rangle=\langle\delta \theta(\omega)\rangle=0$ and that $\langle\delta \varepsilon(\omega)\rangle=0$. The spectral densities of $\delta \varphi(\omega)$, $\delta \theta(\omega)$ and their cross-correlations can all be expressed in terms of the current noise spectral density. We have $S_{\theta \theta}(\omega)=\left(\Delta_{0}^{2} / \omega^{2}\right) S_{\varphi \varphi}(\omega), S_{\varphi \theta}(\omega)=-i\left(\Delta_{0} / \omega\right) S_{\varphi \varphi}(\omega)$, and

$$
S_{\varphi \varphi}(\omega)=\frac{e^{2}}{\hbar^{2}}\left(\frac{C_{0}}{C_{i}}\right)^{2} \frac{\omega^{2}|Z(\omega)|^{2}}{\left(\omega^{2}-\Omega_{0}^{2}\right)^{2}} S_{I I}(\omega)
$$

We will relate the reduction of the persistent current to $S_{\varphi \varphi}(\omega)$ in Sec. III. While the spectral densities for $\delta \varphi$ and $\delta \theta$ are finite at zero frequency, the spectral density of the global phase $\delta \chi$ is not. We have $S_{\chi \chi}(\omega)=$ $\left(\Omega_{0}^{2} \varepsilon_{0}^{2} / \omega^{4}\right) S_{\varphi \varphi}(\omega)$, see Eq. (28), therefore it exhibits a $\omega^{-2}$-pole in the vicinity of $\omega=0$ for finite temperatures

$$
S_{\chi \chi}(\omega) \sim 2 \pi \alpha \frac{2 k T}{\hbar} \frac{\varepsilon_{0}^{2}}{\Omega_{0}^{2}} \frac{1}{\omega^{2}}, \quad(\omega \rightarrow 0),
$$

whereas in the quantum limit $T=0$ the order of the pole is reduced by one,

$$
S_{\chi \chi}(\omega) \sim 2 \pi \alpha \frac{\varepsilon_{0}^{2}}{\Omega_{0}^{2}} \frac{1}{|\omega|}, \quad(\omega \rightarrow 0),
$$

see Fig. 3. These two last results do not affect the persistent current, but they are of great importance in the discussion of the rates of phase diffusion in Sec. IV]. 


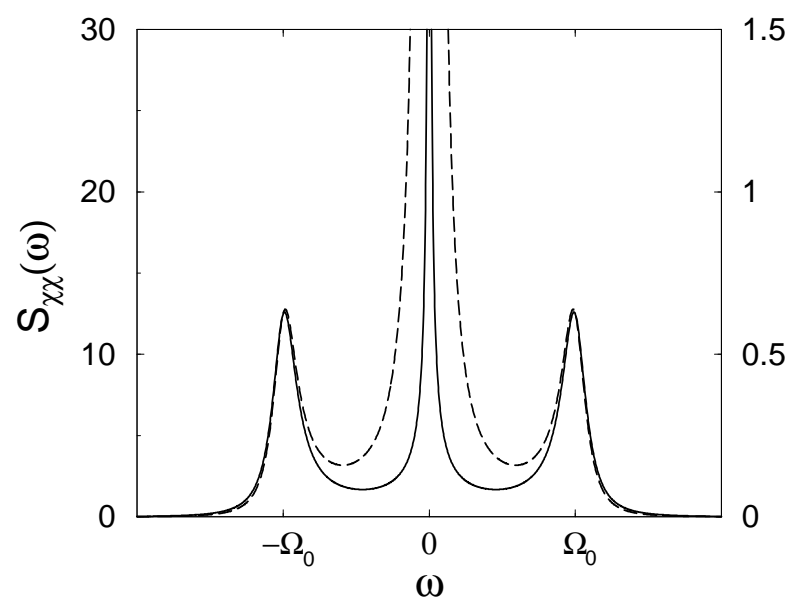

FIG. 3. The spectral function $S_{\chi \chi}(\omega)$ at finite temperature $k T=10 \hbar \Omega_{0}$ (solid line) and at zero temperature (dashed line). The left $y$-axis carries the scale for the finite temperature curve, the right one carries the scale for the zero temperature curve. The parameters are the same as in Fig. 4 in addition $\varepsilon_{0}=\Omega_{0} / \sqrt{2}$.

\section{E. Weak coupling}

Within the approach out-lined above, we can expect to reproduce the exact result for the reduction of the persistent current only in the limit of weak coupling between the ring-dot system and the external circuit. This means that the potential fluctuations of the system and the ex- ternal circuit are only weakly coupled. Thus weak coupling between the ring and the external circuit is achieved by letting the capacitances coupling the two systems become very small $28 C_{1}, C_{2} \rightarrow 0$, for a fixed resistance $R$. This entails $C_{0} \rightarrow 0$, thus the coupling strength $\alpha$ between the ring and the external circuit, Eq. (24), is small against 1 . Note that in this approximation the RC-time $\tau_{R C}$ becomes very small as well.

In the following paragraph, we calculate the poles of $Z(\omega)$, in the approximation $C_{0} \rightarrow 0$. The small parameters in this case are $\tau_{R C} \propto C_{0}$ and $\Gamma \propto C_{0}^{2}$. The equation $[Z(\omega)]^{-1}=0$ has one solution behaving as $C_{0}^{-1}$. To leading order in $C_{0}$, it reads

$$
\omega_{3}=-\frac{i}{\tau_{R C}}
$$

Thus in the weak coupling limit charge relaxation across the mesoscopic system becomes instantaneous. The other two solutions, $\omega_{ \pm}$, are of zeroth order in $C_{0}$. Up to and including corrections of order $C_{0}^{2}$, they are

$$
\omega_{ \pm}= \pm \Omega_{0}-i \frac{\Gamma}{2}
$$

with a relaxation rate $\Gamma$ introduced in Eq. (23). The rate $\Gamma$ describes the relaxation of perturbations with a frequency close to the eigenfrequency of the isolated ringdot system. In Sec. IV it is shown that $\Gamma$ is also related to a phase diffusion time. The denominator in Eq. (34) can now be written as

$$
\frac{\left(\omega^{2}-\Omega_{0}^{2}\right)^{2}}{|Z(\omega)|^{2}}=\frac{\tau_{R C}^{2}}{R^{2}}\left(\omega^{2}+\frac{1}{\tau_{R C}^{2}}\right)\left[\left(\omega-\Omega_{0}\right)^{2}+(\Gamma / 2)^{2}\right]\left[\left(\omega+\Omega_{0}\right)^{2}+(\Gamma / 2)^{2}\right] .
$$

For frequencies, $\omega \tau_{R C} \ll 1$, the denominator takes the form

$$
\frac{\left(\omega^{2}-\Omega_{0}^{2}\right)^{2}}{|Z(\omega)|^{2}}=\frac{1}{R^{2}}\left[\left(\omega-\Omega_{0}\right)^{2}+(\Gamma / 2)^{2}\right]\left[\left(\omega+\Omega_{0}\right)^{2}+(\Gamma / 2)^{2}\right] .
$$

Since in the quantum limit it is always necessary to introduce a cut-off, Eq. (40) is valid over the entire range of frequencies of interest, if only $1 / \tau_{R C}$ is larger than the cut-off frequency. The spectral density $S_{\varphi \varphi}(\omega)$, Eq. (34), reads therefore

$$
S_{\varphi \varphi}(\omega)=\frac{2 \pi \alpha \omega^{3} \operatorname{coth} \frac{\hbar \omega}{2 k T}}{\left[\left(\omega-\Omega_{0}\right)^{2}+(\Gamma / 2)^{2}\right]\left[\left(\omega+\Omega_{0}\right)^{2}+(\Gamma / 2)^{2}\right]},
$$

where the coupling strength $\alpha$ between the ring and the external circuit is defined in Eq. (24). Note that $S_{\varphi \varphi}(\omega)$ goes to zero at the origin $\omega=0$ like $|\omega|^{3}$ in the extreme quantum case $(T=0)$, and like $\omega^{2}$ for finite temperatures, see Fig. 4. For $|\omega| \gg \Omega_{0}$, it behaves like $|\omega|^{-1}$ for small temperatures and like $\omega^{-2}$ for large temperatures. Analogous formulæ hold for $S_{\theta \theta}(\omega)$ and for $S_{\varphi \theta}(\omega)$. We mention that the spectral density $S_{\theta \theta}(\omega)$ behaves for small $\omega$ like $|\omega|$ at $T=0$ and goes to a constant for finite $T$. All the three spectral densities exhibit peaks of width $\Gamma$ at $\pm \Omega_{0}$. 


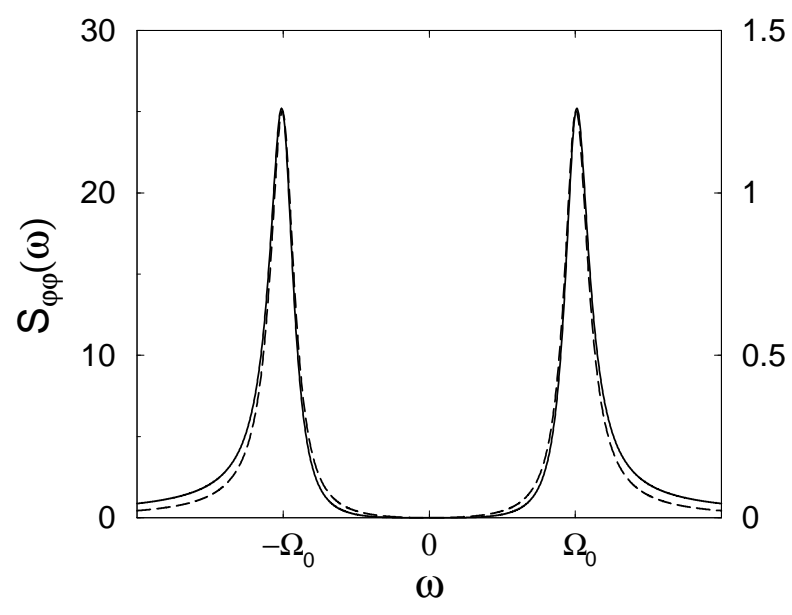

FIG. 4. The spectral function $S_{\varphi \varphi}(\omega)$ at finite temperature $k T=10 \hbar \Omega_{0}$ (solid line) and at zero temperature (dashed line). The left $y$-axis carries the scale for the finite temperature curve, the right one carries the scale for the zero temperature curve. The parameters are $R=5 R_{K}, C_{0}=0.1 C_{i}$, $\Delta_{0}=0.5 \Omega_{0}$ and $\Gamma=0.2 \Omega_{0}$.
The spectral density of the global phase $S_{\chi \chi}(\omega)$, Fig. 33, exhibits peaks at $\omega= \pm \Omega_{0}$ as well. More important, however, is the fact that it has a pole at $\omega=0$, as already pointed out in the previous section.

\section{PERSISTENT CURRENT}

In the following, we calculate the persistent current as a quantum and statistical average of the operator of the circulating current

$$
\hat{I}_{c}=\left(\begin{array}{cc}
0 & \mathcal{J} \\
\mathcal{J}^{*} & 0
\end{array}\right)
$$

where $\mathcal{J}$ is given by

$$
\mathcal{J}=-\frac{e}{\hbar}\left(t_{L}^{2}+t_{R}^{2} \pm 2 t_{L} t_{R} \cos \frac{2 \pi \Phi}{\Phi_{0}}\right)^{-1 / 2}\left\{ \pm t_{L} t_{R} \sin \frac{2 \pi \Phi}{\Phi_{0}}-i\left[\frac{C_{R}}{C_{i}} t_{L}^{2}-\frac{C_{L}}{C_{i}} t_{R}^{2} \pm \frac{C_{R}-C_{L}}{C_{i}} t_{L} t_{R} \cos \frac{2 \pi \Phi}{\Phi_{0}}\right]\right\}
$$

Here the first term is a pure particle current contribution, whereas the second term is a consequence of interactions. For the average persistent current of interest here, it is only the first term which contributes. A derivation of Eq. (43) and a discussion of the relationship between $I_{c}$ and the magnetization is given in Appendix B.

The expectation value of the persistent current for the state given in Eq. (13) reads

$$
I(t) \equiv\left\langle\psi(t)\left|\hat{I}_{c}\right| \psi(t)\right\rangle=\frac{1}{2} \operatorname{Re} \mathcal{J} \sin \theta e^{-i \varphi} .
$$

We are, however, interested in the statistically averaged of the persistent current $\langle I(t)\rangle$, given by

$$
\left\langle\left\langle\psi(t)\left|\hat{I}_{c}\right| \psi(t)\right\rangle\right\rangle=\frac{1}{2} \operatorname{Re}\left(\mathcal{J}\left\langle\sin \theta e^{-i \varphi}\right\rangle\right),
$$

where the double bracket indicates a quantum and statistical average. Therefore, we have to calculate the correlator $\left\langle\sin \theta e^{-i \varphi}\right\rangle$. First, we observe that there are no correlations between $\delta \varphi$ and $\delta \theta$,

$$
\langle\delta \varphi(t) \delta \theta(t)\rangle=0
$$

as the spectral density $S_{\varphi \theta}(\omega)$ is an odd function of $\omega$. Thus we have

$$
\left\langle\sin \theta e^{-i \varphi}\right\rangle=\langle\sin \theta\rangle\left\langle e^{-i \varphi}\right\rangle,
$$

to second order in $\delta I$, that is, the probability distributions for $\varphi$ and $\theta$ are decoupled up to second order in $\delta I$. Second, it can be shown that the second order corrections to $\varphi$ and $\theta$ vanish on the average. Finally, we assume that the correlations in $\delta \varphi$ are Gaussian, which allows us to write

$$
\left\langle e^{-i \varphi(t)}\right\rangle=e^{-i \varphi_{0}}\left\langle e^{-i \delta \varphi(t)}\right\rangle=\left\langle\exp \left(-\frac{\delta \varphi^{2}(t)}{2}\right)\right\rangle,
$$

where we have used that $\varphi_{0}=0$. In the weak coupling limit, see Sec. IIE, and in the extreme quantum limit, $T=0$, we find for the time averaged mean square fluctuations

$$
\left\langle\delta \varphi^{2}(t)\right\rangle=\int_{0}^{\omega_{c}} \frac{d \omega}{\pi} S_{\varphi \varphi}(\omega) \approx 2 \alpha \ln \frac{\omega_{c}}{\Omega_{0}}
$$

where the cut-off frequency $\omega_{c}$ is taken to be larger than all frequency scales (except $\tau_{R C}^{-1}$ ), and $\alpha$ is the coupling strength between ring and external circuit, see Eq. (24). In the limit $\omega_{c} \gg \Omega_{0}$, we can neglect $\left\langle\delta \theta^{2}(t)\right\rangle=$ $\int_{0}^{\omega_{c}}(d \omega / \pi) S_{\theta \theta}(\omega)$ against $\left\langle\delta \varphi^{2}(t)\right\rangle$. We insert $\left\langle\delta \varphi^{2}(t)\right\rangle$ and $\sin \theta_{0}=\Delta_{0} / \Omega_{0}$ into $\left\langle\sin \theta e^{-i \varphi}\right\rangle$, and observe that

$$
\frac{1}{2} \operatorname{Re} \mathcal{J}=\frac{\hbar c}{2} \frac{\partial \Delta_{0}}{\partial \Phi} .
$$

Put together, we obtain the noise averaged current in the ring

$$
\langle I(t)\rangle=-\frac{\hbar c}{2} \frac{\partial \Delta_{0}}{\partial \Phi} \frac{\Delta_{0}}{\Omega_{0}}\left(\frac{\Omega_{0}}{\omega_{c}}\right)^{\alpha}
$$


Eq. (51) is a key result of this work. For $\alpha \ll 1$, corresponding to weak coupling between the ring and the external circuit, the power law for the persistent current obtained in Eq. (51), as well as the exponent $\alpha$, Eq. (24), are the same as the one obtained when the external circuit is treated quantum mechanically as well. In a recent work, the authors in collaboration with Ponomarenko have shown that if the external circuit is represented by a transmission line, the persistent current at resonance, $\varepsilon=0$, has for $\alpha<1$ the power law behavior

$$
I(\varepsilon=0) \propto\left(\frac{\Delta_{0}}{\omega_{c}}\right)^{\frac{\alpha}{1-\alpha}} .
$$

For a very small coupling parameter, $\alpha \ll 1$, the Bethe ansatz result Eq. (52), goes over to the power law of Eq. (51). Thus the simplified discussion presented here leads at least in the weak coupling limit to the same result as the one obtained in Ref. 1.

We emphasize that the persistent current is a property of the ground state of a system. In our case, the persistent current is, however, carried by only a part of the system. Due to the coupling to the external circuit this subsystem is subject to fluctuations which even at zero temperatures suppress the persistent current. If we keep the capacitances fixed, Eqs. (51) and (52) give a persistent current which decreases with increasing external resistance $R$. The spectral density of the voltage fluctuations across the ring dot system increases with $R$ (see Eq. (6)). We next characterize the fluctuations of the ring-dot subsystem in more detail.

\section{PHASE DIFFUSION TIMES}

In this work we are concerned with the effect of an external circuit on the ground state properties of the system and in particular on the persistent current of the ringdot subsystem. In transport experiments which reveal phase-coherent contributions or in experiments which investigate the evolution of an initial state, we can describe the effect of an environment with the help of dephasing rates. On the other hand, it is not a priori clear that the coherence properties of the ground state of a system, can be equally expressed in terms of dephasing rates. After all the persistent current investigated here exists quite independently on how long we let the system evolve. Here we consider the state in the ring and investigate its evolution away from an initial state. Below we show that this evolution is diffusive at least for times short enough such that we remain within the limit of validity of the calculation.
We have seen in Sec. IIE that the dynamics of the ring strongly affects the spectral densities $S_{\varphi \varphi}(\omega)$ and $S_{\theta \theta}(\omega)$ in the vicinity of the characteristic frequency $\Omega_{0}$. The characteristic frequency describes the free dynamics of a ring which is disconnected from the external circuit, described by the Hamiltonian $\hat{H}_{\text {ring }}$, Eq. (9), without the time dependent terms $\delta \varepsilon(t)$ and $\nu(t)$,

$$
\hat{H}_{0}=\frac{\hbar \varepsilon_{0}}{2} \sigma_{z}-\frac{\hbar \Delta_{0}}{2} \sigma_{x} .
$$

This Hamiltonian can be viewed as describing a spin in a magnetic field of strength proportional to $\left(\varepsilon_{0}^{2}+\Delta_{0}^{2}\right)^{1 / 2}=$ $\Omega_{0}$, forming an angle $\theta_{0}$ with the $z$-axis. The angle $\theta_{0}$ has been defined in Sec. IIB and is related to $\varepsilon_{0}$ and $\Delta_{0}$ by $\cot \theta_{0}=-\varepsilon_{0} / \Delta_{0}$. The eigenstates of the time independent Hamiltonian $\hat{H}_{0}$ are the ground state $\psi_{-}=\left(\cos \theta_{0} / 2, \sin \theta_{0} / 2\right)$, with eigenvalue $-\hbar \Omega_{0} / 2$, and the excited state $\psi_{+}=\left(-\sin \theta_{0} / 2, \cos \theta_{0} / 2\right)$ with eigenvalue $\hbar \Omega_{0} / 2$. The evolution due to $\hat{H}_{0}$ of a spin prepared in a state which is not an eigenstate corresponds to a rotation of the expectation value of the spin about the direction of the magnetic field with a frequency $\Omega_{0}$. Let us now return to the full problem in which polarization fluctuations modify the free evolution of the decoupled system. In the weak coupling limit, we are interested in the time evolution which is long compared to $\Omega_{0}^{-1}$. Therefore, we switch to the "rotating frame" picture, where the wave function $\psi(t)$ of the ring, Eq. (13), reads $\psi_{R}(t) \equiv \exp \left(i \hat{H}_{0} t / \hbar\right) \psi(t)$. We consider the projection $c_{ \pm}(t) \equiv\left\langle\psi_{ \pm} \mid \psi_{R}(t)\right\rangle$ of the wave function $\psi_{R}(t)$ onto the states $\psi_{ \pm}$. If the state $\psi(t)$ evolves under the influence of the time independent Hamiltonian $\hat{H}_{0}$, the projections $c_{ \pm}(t)$ are constant in time. The moduli of the projections, averaged over the noise, $\left\langle\left|c_{ \pm}(t)\right|^{2}\right\rangle$ are also independent of time if the evolution is determined by $\hat{H}_{\text {ring }}$ which includes the fluctuating potential. The phase of the projections $c_{ \pm}(t)$, however, shows diffusive behavior. On the average, we have $\left\langle\left|c_{ \pm}(t)-c_{ \pm}(0)\right|^{2}\right\rangle \sim t / \tau_{ \pm}$for sufficiently long times $t$. We shall see below that $\tau_{-}$is related to the phase diffusion of the global phase $\chi$, whereas $\tau_{+}$is related to the diffusion of the internal phases $\varphi$ and $\theta$, see Eq. (13). It is a known feature of two-leve-systems that they exhibit two distinct dephasing times.29 The difference between $\tau_{-}$and $\tau_{+}$is particularly pronounced in the low temperature limit. The phase breaking time $\tau_{-}$ diverges at zero temperature, whereas the time $\tau_{+}$saturates to a finite value for temperatures $k T$ below $\hbar \Omega_{0}$.

We expand the wave function $\psi_{R}$ to first order in $\delta \varepsilon$, that is, to first order in $\delta \varphi, \delta \theta$ and $\delta \chi$,

$$
\psi_{R}(t)=\left[1+i\left(\frac{\delta \chi(t)}{2}-\frac{\varepsilon_{0}}{\Omega_{0}} \frac{\delta \varphi(t)}{2}\right)\right] \psi_{-}+\left[\frac{\delta \theta(t)}{2}-i \frac{\Delta_{0}}{\Omega_{0}} \frac{\delta \varphi(t)}{2}\right] e^{i \Omega_{0} t} \psi_{+}
$$

From this expression, we immediately obtain the projections $c_{-}(t)=1+i\left[\delta \chi(t) / 2-\left(\varepsilon_{0} / \Omega_{0}\right) \delta \varphi(t) / 2\right]$ and $c_{+}(t)=$ $1 / 2\left(\delta \theta(t)-i\left(\Delta_{0} / \Omega_{0}\right) \delta \varphi(t)\right) \exp \left(i \Omega_{0} t\right)$. As the averages $\left\langle\delta \varphi^{2}(t)\right\rangle,\left\langle\delta \theta^{2}(t)\right\rangle$ and $\left\langle\delta \chi^{2}(t)\right\rangle$ as well as all the "crossed" 
averages of the type $\langle\delta \varphi(t) \delta \theta(t)\rangle$ are constant in $t$, the moduli $\left\langle\left|c_{ \pm}(t)\right|^{2}\right\rangle$ are indeed independent of time. The mean squared displacement of the projections $c_{ \pm}(t)$ read respectively

$$
\begin{aligned}
\left\langle\left|c_{-}(t)-c_{-}(0)\right|^{2}\right\rangle & =\int \frac{d \omega}{2 \pi} \sin ^{2} \frac{\omega t}{2}\left[S_{\chi \chi}(\omega)+\frac{\varepsilon_{0}^{2}}{\Omega_{0}^{2}} S_{\varphi \varphi}(\omega)\right], \\
\left\langle\left|c_{+}(t)-c_{+}(0)\right|^{2}\right\rangle & =\int \frac{d \omega}{2 \pi} \sin ^{2} \frac{\omega t}{2}\left[S_{\theta \theta}\left(\omega+\Omega_{0}\right)+\frac{\Delta_{0}^{2}}{\Omega_{0}^{2}} S_{\varphi \varphi}\left(\omega+\Omega_{0}\right)\right] .
\end{aligned}
$$

The long time behavior of Eq. (55) is dominated by the frequencies near $\omega=0$. The spectral density $S_{\varphi \varphi}(\omega)$ vanishes like $\omega^{2}$ for finite temperatures or even like $|\omega|^{3}$ in the zero temperature limit. The spectral density $S_{\chi \chi}(\omega)$, however has a pole at $\omega=0$. For finite temperatures the pole is of order $\omega^{-2}$, see Eq. (35), entailing a long time behavior of the type $\left\langle\left|c_{-}(t)-c_{-}(0)\right|^{2}\right\rangle \sim t / \tau_{-}$, with a dephasing time

$$
\tau_{-}=\frac{\hbar}{2 \pi \alpha k T} \frac{\Omega_{0}^{2}}{\varepsilon_{0}^{2}}
$$

We stress again that $\tau_{-}$determines the diffusion time of the global phase $\chi$ as it is given in terms of the spectral function $S_{\chi \chi}(\omega)$. In the quantum limit $T=0$, the phase diffusion time $\tau_{-}$diverges. Furthermore, it follows from Eq. (57) that $\tau_{-}$is tunable, since $\varepsilon_{0}$ may be varied by an external DC-bias. In particular, at resonance $\varepsilon_{0}=0$, the phase diffusion time $\tau_{-}$diverges for any temperature.

The long time behavior of Eq. (56), on the other hand, is determined by the frequencies near $\Omega_{0}$. In the vicinity of this characteristic frequency, $S_{\varphi \varphi}\left(\Omega_{0}+\omega\right)$ as well as $S_{\theta \theta}\left(\Omega_{0}+\omega\right)$ show a $\omega^{-2}$ behavior at finite as well as at zero temperature, which is cut off by the relaxation rate $\Gamma$, defined in Eq. (23) at very small frequencies $\omega \sim \Gamma \ll \Omega_{0}$. In summary, we have, for $|\omega| \ll \Omega_{0}$

$$
S_{\varphi \varphi}\left(\Omega_{0}+\omega\right) \approx \frac{2 \pi \alpha \Omega_{0} \operatorname{coth} \frac{\hbar \Omega_{0}}{2 k T}}{\omega^{2}+(\Gamma / 2)^{2}}
$$

and $S_{\theta \theta}\left(\Omega_{0}+\omega\right) \approx\left(\Delta_{0}^{2} / \Omega_{0}^{2}\right) S_{\varphi \varphi}\left(\Omega_{0}+\omega\right)$. The time evolution of Eq. (56) for times much larger than the inverse of the characteristic frequency $\Omega_{0}$, yet smaller than the inverse of the relaxation rate $\Gamma$, is therefore linear in time with a characteristic time $\tau_{+}$, where

$$
\tau_{+}=\frac{1}{\Gamma} \tanh \frac{\hbar \Omega_{0}}{2 k T}
$$

Note that Eq. (59) holds for finite temperatures as well as in the quantum limit. The phase diffusion time $\tau_{+}$is inversely proportional to $T$ at high temperatures,

$$
\tau_{+}=\frac{1}{\Gamma} \frac{\hbar \Omega_{0}}{2 k T}, \quad\left(k T \gg \hbar \Omega_{0}\right),
$$

just as the other characteristic time $\tau_{-}$. In the low temperature or quantum limit, however, it saturates to a value

$$
\tau_{+}^{(0)}=\frac{1}{\Gamma}, \quad\left(k T \ll \hbar \Omega_{0}\right),
$$

see also Fig. 5. The crossover from high temperature behavior to the quantum limit behavior takes place at $k T \sim \hbar \Omega_{0}$. We point out that $\tau_{+}$is related to the spectral densities $S_{\varphi \varphi}(\omega)$ and $S_{\theta \theta}(\omega)$ of the internal phases $\varphi$ and $\theta$. This indicates that there is a relation between dephasing (at finite temperature) and the reduction of the persistent current at zero temperature which is determined by $S_{\varphi \varphi}(\omega)$.

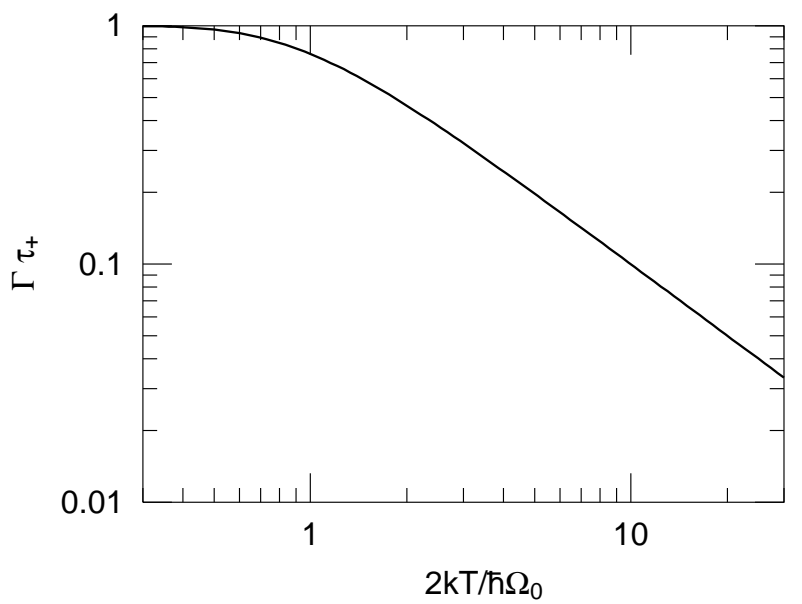

FIG. 5. The characteristic phase diffusion time $\tau_{+}$normalized by the decay rate $\Gamma$, defined in Eq. 23), as a function of $2 k T / \hbar \Omega_{0}$ in a logarithmic scale. For high temperatures, $\tau_{+}$is inversely proportional to the temperature $T$. For low temperatures, $k T \ll \hbar \Omega_{0}$, it saturates at a value $\tau_{+}=\Gamma^{-1}$.

We point out that Eq. (55) and Eq. (56) do not hold for arbitrarily long times. In reality the mean square displacements $\left\langle\left|c_{ \pm}(t)-c_{ \pm}(0)\right|^{2}\right\rangle$ are bounded since the wave function $\psi_{R}(t)$ is normalized to 1 . The fact that $\left\langle\left|c_{-}(t)-c_{-}(0)\right|^{2}\right\rangle$, Eq. (55), grows without bounds is an artifact of the linearization of Eqs. (14)-(16) and Eq. (20).

In summary, we find two characteristic phase diffusion times $\tau_{-}$and $\tau_{+}$, related to the projection of the equilibrium state $\psi(t)$ onto the ground state and the excited state. The time $\tau_{-}$, associated with the projection on the ground state, is related to the loss of coherence in the global phase $\chi$ and diverges as the temperature goes to zero. The time $\tau_{+}$, on the other hand, characterizes the loss of coherence in the internal phases $\varphi$ and $\theta$, and sat- 
urates to a finite value in the limit of zero temperature. This indicates that even at zero temperature, coupling to the external circuit causes excitations of the ring into the excited state. In the zero-temperature limit these excitations still decay in a finite time.

\section{CONCLUSION}

We have investigated the persistent current in a normal metal ring coupled to a resistive external circuit using a Langevin equation approach. We have shown that the quantum fluctuations in the external circuit suppress the persistent current at zero temperature, thus confirming an earlier Bethe ansatz result.1 Within the same framework, but at finite temperature, we have derived two dephasing times $\tau_{-}$and $\tau_{+}$, which are the phase diffusion times of the projections of the wave function of the ring to ground state and the excited state, respectively, of a ring which is disconnected from the external circuit. We show that $\tau_{+}$is related to the spectral densities of the "internal" phases $\varphi$ and $\theta$ of the wave function of the ring, which are also responsible for the reduction of the persistent current. While $\tau_{-}$diverges in the zero temperature limit, $\tau_{+}$saturates to a finite value.

\section{APPENDIX A: COULOMB INTERACTIONS AND DISPLACEMENT CURRENTS}

In this Appendix, we briefly discuss the electrical coupling of the ring and the external circuit. The fluctuations of the charge in the ring and the external circuit are not arbitrary but are connected by the flow of a displacement current between the ring and the external circuit. We can express the coupling between the ring and external circuit in terms of the displacement current. Since the coupling between these subsystems is crucial, we present first a discussion of the displacement currents.

In our model the charges and potentials are related via

$$
\begin{aligned}
Q_{0} & =C_{1}\left(V_{0}-U_{d}\right) \\
Q_{d} & =C_{1}\left(U_{d}-V_{0}\right)+C_{i}\left(U_{d}-U_{a}\right) \\
Q_{a} & =C_{2}\left(U_{a}-V_{\infty}\right)+C_{i}\left(U_{a}-U_{d}\right) \\
Q_{\infty} & =C_{2}\left(V_{\infty}-U_{a}\right)
\end{aligned}
$$

where we have introduced the parallel capacitance $C_{i} \equiv$ $C_{L}+C_{R}$. In the following, we shall also need the external (serial) capacitance $C_{e}^{-1} \equiv C_{1}^{-1}+C_{2}^{-1}$, as well as the parallel and the serial total capacitances $C \equiv C_{i}+C_{e}$ and $C_{0}^{-1} \equiv C_{i}^{-1}+C_{e}^{-1}$. The whole structure, that is, the ring together with the external circuit is charge neutral. As a matter of fact, it follows from Eqs. (A1)-(A4) that $Q_{0}+Q_{d}+Q_{a}+Q_{\infty}=0$. The ring and the external circuit taken separately, however, do not need to be neutral. The total charge of the ring $Q_{d}+Q_{a}$ is balanced by the charge on the external capacitors $C_{1}$ and $C_{2}$,
$Q_{0}+Q_{\infty}=-\left(Q_{d}+Q_{a}\right)$, as follows from Eqs. (A1)-( $\left.\mathrm{A} 4\right)$. As the ring and the external circuit do not exchange particles, the charges on the ring and in the external circuit are conserved, and it is more convenient to consider the deviations from a reference state. We denote these deviations by $\delta Q_{d}$ on the dot and by $\delta Q_{a}$ on the arm, and similarly by $\delta Q_{0}$ and $\delta Q_{\infty}$ for the external circuit. They obey the relations $\delta Q_{d}+\delta Q_{a}=\delta Q_{0}+\delta Q_{\infty}=0$. For time derivatives, it is of course irrelevant whether we consider total charges or deviations.

The current flowing out from the capacitor $C_{1}$ is a pure displacement current

$$
I_{1}=-\dot{Q}_{0}=C_{1} \frac{\partial}{\partial t}\left(U_{d}-V_{0}\right),
$$

whereas the currents flowing through the right and the left junction, respectively, are particle currents $I_{L / R}^{p}$ augmented by displacement currents

$$
\begin{aligned}
& I_{L}=I_{L}^{p}+C_{L} \frac{\partial}{\partial t}\left(U_{d}-U_{a}\right), \\
& I_{R}=I_{R}^{p}+C_{R} \frac{\partial}{\partial t}\left(U_{d}-U_{a}\right) .
\end{aligned}
$$

The particle currents are related to the charge on the dot by $I_{L}^{p}+I_{R}^{p}=-\dot{Q}_{d}$. At each node we have $I_{1}+I_{L}+I_{R}=$ $I_{2}-\left(I_{L}+I_{R}\right)=0$, and thus $I_{1}+I_{2}=0$. These equations correspond to the law of current conservation at the nodes of an electrical network.

We can express all electrical quantities of interest in terms of the external bias $\delta V \equiv V_{0}-V_{\infty}$ and the charge deviation, $\delta Q_{d}$. We combine Eqs. (A2) and (A3) and the condition $\delta Q_{d}+\delta Q_{a}=0$ to obtain the potential difference inside the ring

$$
U_{d}-U_{a}=\frac{C_{e}}{C} \delta V+\frac{\delta Q_{d}}{C} .
$$

The charge deviation $\delta Q_{0}$ on the capacitor $C_{1}$ is found from Eqs. (A1), (A4) and (A8),

$$
\delta Q_{0}=C_{0} \delta V-\frac{C_{0}}{C_{i}} \delta Q_{d}
$$

where we have used the identity

$$
\frac{C_{e}}{C}=\frac{C_{0}}{C_{i}},
$$

whence we obtain for the current flowing in the external circuit

$$
I_{1}=\frac{C_{0}}{C_{i}} \dot{Q}_{d}-C_{0} \delta \dot{V} .
$$

In the circuit containing a current noise source, see Fig. 22, the current $I_{1}$ flowing out of the circuit augmented by the current $\delta I$ coming from the noise source must equal the current through the resistor $I=\delta V / R$, namely

$$
\delta V / R=I_{1}+\delta I \text {. }
$$

Together with Eq. A11), this gives Eq. (1). 


\section{APPENDIX B: THE CIRCULATING CURRENT}

The equilibrium persistent current $I$ is a quantum and statistical average which can be obtained from the derivative of the free energy $I=-c \partial F / \partial \Phi$. Some discussion is required, if we are, as in Ref. 1 concerned with current fluctuations. Naively, we might want to investigate the fluctuations of the persistent current by considering the second order derivative of the thermodynamic potential. However, such a procedure works only if the observable of interest commutes with the Hamiltonian. Moreover, as pointed out above, the true and physically relevant currents is the total current (particle current plus displacement current). Whereas the displacement current needs not to be considered as long as we are interested in average quantities only, this is not true, if we consider fluctuations of the current. For the model considered here, we can derive expressions for the current operators which are particle operators weighted according to the distribution of the geometrical capacitances of the system.

Consider first for a moment the electrically isolated loop. In this case the capacitances to the exterior circuit are $C_{1}=C_{2}=0$. From Eq. (A2) we find $Q_{d}=C_{i}\left(U_{d}-\right.$ $U_{a}$ ) and thus we can also write the currents through the left and right junctions, Eqs. (A6) and (A7), in terms of the charge on the dot as, $I_{L}=I_{L}^{p}+\left(C_{L} / C_{i}\right) d Q_{d} / d t$ and $I_{R}=I_{R}^{p}+\left(C_{R} / C_{i}\right) d Q_{d} / d t$. Using particle conservation, $I_{L}^{p}+I_{R}^{p}-d Q_{d} / d t=0$, to eliminate the time-derivative of the charge we find immediately that the current circulating in this loop is, $\hat{I}_{c} \equiv \hat{I}_{L}=-\hat{I}_{R}$ with

$$
\hat{I}_{c}=\frac{C_{R} \hat{I}_{L}^{p}-C_{L} \hat{I}_{R}^{p}}{C_{i}}
$$

where $C_{i}=C_{L}+C_{R}$. The circulating current is thus in general not determined by the particle currents but by an average of these currents weighted according to the Coulomb interaction (capacitance ratios). Expressions of this type are familiar form the dynamic transport through double barriers, but seem to be novel for persistent currents. We emphasize that Eq. (B1) does not mean that the particle currents can now be calculated from the non-interacting problem. The dynamic particle currents depend on the self-consistent potential distribution. For an illustration of this point we refer the reader to Ref. 30 where dynamic current noise spectra for double barriers are compared based on calculations using the particle currents of the non-interacting problem and calculations using the particle currents of the interacting problem.

Next let us consider the system coupled to the external circuit. In this case $C_{1}>0$ and $C_{2}>0$. We can proceed as above. We first express the time derivative of potential difference $U_{d}-U_{a}$ in terms of the time derivative of the charge on the dot and the current
$I_{1}=d Q_{0} / d t=C_{1} d\left(V_{0}-U_{d}\right) / d t$ (see Eq. A1). Using current conservation, $I_{1}=I_{L}+I_{R}$ and particle conservation $I_{L}^{p}+I_{R}^{p}-d Q_{d} / d t=0$ we can again eliminate the time derivative of the charge $Q_{d}$ and find for the currents through the left and right barrier,

$$
\begin{gathered}
\hat{I}_{L}=\hat{I}_{c}+\left(C_{L} / C_{i}\right) I_{1}, \\
\hat{I}_{R}=-\hat{I}_{c}+\left(C_{R} / C_{i}\right) I_{1},
\end{gathered}
$$

with $\hat{I}_{c}$ as given in Eq. (B1). The current $I_{1}$, which can be induced with the help of the external circuit, is divided onto the two branches of the ring-dot sample also according to a capacitance ratio.

What is observed in a measurement of the magnetization? It is important to note, that the external circuit (see Fig. 1) also forms a current loop and depending on the geometry of the circuit also contributes to the fluctuation of the magnetization. Suppose, the circuit of Fig. 1 is a planar structure in the $x$-y-plane. Let the ring with the in-line dot enclose an area $A_{r}$ and let the external circuit (excluding the ring) enclose an area $A_{e}$. For this circuit the magnetic moment can be viewed as being generated by a current $I_{L}$ enclosing the area $A_{1}$ and by a current $I_{1}$ enclosing the area $A_{e}$. The magnetic moment is thus $m=(1 / c)\left(I_{L} A_{r}-I_{1} A_{e}\right)$. Of course the topology of the current distribution matters: If we consider the external circuit to be above the ring dot structure instead of below as shown in Fig. 1, the magnetization is $m=(1 / c)\left(-I_{R} A_{r}+I_{1} A_{e}\right)$. In these two circuits the external circuit gives magnetization contribution into opposite directions. The average magnetization of the two circuits is just $m=(1 / c) I_{c} A_{e}$. A third circuit which permits to investigate the magnetization of the ring and the external circuit separately is a structure in which the ring lies in the $x-y$-plane and the external circuit say in the $x$-z-plane. Then the magnetization is $m_{z}=(1 / c) I_{c} A_{r}$ and $m_{y}=(1 / c) I_{1} A_{e} . \quad I_{c}$ appears as the most natural generalization of the equilibrium persistent current. In Ref. 11 we have investigated the fluctuations of $I_{c}$.

All the above expressions are based on linear relations between currents, charges and potentials. All the above expressions are thus valid also for operators. We now present a specific expression for the operator of the circulating current used in Ref. 1. Let us consider the particle current operators $\hat{I}_{L}^{p}$ through the left and $\hat{I}_{R}^{p}$ through the right tunnel barrier. In terms of the Hamilton operators $\hat{H}_{L}$ and $\hat{H}_{R}$ responsible for the tunneling across the left and the right tunnel barrier, respectively, they read $\hat{I}_{L / R}^{p}=-i / \hbar\left[\hat{H}_{L / R}, \hat{Q}_{d}\right]$. In other words, they are equal to the decrease of the charge on the dot per unit of time through the right and left junction. For the two-level system, in a basis in which the Hamiltonian is real (see Eq. (9)) we have 


$$
\begin{aligned}
& \hat{I}_{L}^{p}=-\frac{e}{\hbar} \frac{ \pm t_{L} t_{R} \sin \left(2 \pi \Phi / \Phi_{0}\right) \sigma_{x}+\left[t_{L}^{2} \pm t_{L} t_{R} \cos \left(2 \pi \Phi / \Phi_{0}\right)\right] \sigma_{y}}{\sqrt{t_{L}^{2}+t_{R}^{2} \pm 2 t_{L} t_{R} \cos \left(2 \pi \Phi / \Phi_{0}\right)}} \\
& \hat{I}_{R}^{p}=\frac{e}{\hbar} \frac{ \pm t_{L} t_{R} \sin \left(2 \pi \Phi / \Phi_{0}\right) \sigma_{x}-\left[t_{R}^{2} \pm t_{L} t_{R} \cos \left(2 \pi \Phi / \Phi_{0}\right)\right] \sigma_{y}}{\sqrt{t_{L}^{2}+t_{R}^{2} \pm 2 t_{L} t_{R} \cos \left(2 \pi \Phi / \Phi_{0}\right)}} .
\end{aligned}
$$

Using the particle current operators in Eq. (B1) leads to the operator given by Eq. (42). With the help of this operator we can investigate the average persistent current and the fluctuations (see Eq. (7) of Ref. 1).

${ }^{1}$ P. Cedraschi, V. V. Ponomarenko, and M. Büttiker, Phys. Rev. Lett. 84, 346 (2000).

${ }^{2}$ Details of the calculations leading to the results of Ref. 1 can be found in P. Cedraschi and M. Büttiker, (unpublished) and P. Cedraschi, PhD - thesis, University of Geneva (2000).

${ }^{3}$ F. W. J. Hekking and L. I. Glazman, Phys. Rev. B 55, 6551 (1997).

${ }^{4}$ M. Oshikawa and A. M. Zagoskin, Superlattices and Microstructures, 25, 1177 (1999).

${ }^{5}$ P. Mohanty, E. M. Q. Jariwala, and R. A. Webb, Phys. Rev. Lett. 78, 3366 (1997).

${ }^{6}$ D. S. Golubev and A. D. Zaikin, Phys. Rev. Lett. 81, 1074 (1998).

${ }^{7}$ I. L. Aleiner, B. L. Altshuler, and M. E. Gershenson, Waves in Random Media 9, 201 (1999).

${ }^{8}$ I. L. Aleiner, B. L. Altshuler, and M. E. Gershenson, Phys. Rev. Lett. 82, 3190 (1999).

${ }^{9}$ D. S. Golubev and A. D. Zaikin, Phys. Rev. Lett. 82, 3191 (1999).

${ }^{10}$ V. E. Kravtsov and B. L. Altshuler, Phys. Rev Lett. 84, 33 (2000); Y. Imry, H. Fukuyama, P. Schwab, European Phys. Lett. 47, 608 (1999); A. Zawadowski, J. von Delft, D. C. Ralph, Phys. Rev. Lett. 83, 2632 (1999).

${ }^{11}$ R. Landauer and M. Büttiker, Phys. Rev. Lett. 54, 2049 (1985).

12 M. Büttiker, in SQUID'85, Superconducting Quantum Interference Devices and their Applications, edited by H. D. Hahlbohm and H. Lübbig (Walter de Gruyter, Berlin, New York, 1985), pp. 529-560.

${ }^{13}$ M. Büttiker, Annals of the New York Academy of Sciences 480, 194 (1986).
${ }^{14}$ M. Büttiker, Phys. Rev. B 32, 1846 (1985).

${ }^{15}$ H.-F. Cheung, Y. Gefen, and E. K. Riedel, IBM J. Res. Develop. 32, 359 (1988).

${ }^{16}$ P. A. Mello, Phys. Rev. B 47, 16358 (1993).

${ }^{17}$ M. V. Moskalets, Physica E, 4, 111 (1999).

18 M. T. Liu and C. S. Chu, Phys. Rev. B 61, 7645 (2000); Solid State Commun. 114, 167 (2000).

${ }^{19}$ P. Cedraschi and M. Büttiker, J. Phys.: Condens. Matter 10, 3985 (1998).

${ }^{20}$ M. Büttiker and C. A. Stafford, Phys. Rev. Lett. 76, 495 (1996).

${ }^{21}$ M. Büttiker and C. A. Stafford, in Correlated Fermions and Transport in Mesoscopic Systems, edited by T. Martin, G. Montambaux, and J. Trân Thanh Vân (Editions Frontières, Gif-sur-Yvette, 1996), pp. 491-500.

${ }^{22}$ R. Kotlyar and S. Das Sarma, Phys. Rev. B 55, R10205 (1997).

${ }^{23}$ P. Sandstroem and I. V. Krive, Phys. Rev. B 56, 9255 (1997).

${ }^{24}$ H.-P. Eckle, H. Johannesson, and C. A. Stafford, J. Low Temp. Phys. 118, 475 (2000).

${ }^{25}$ K. Kang and S.-C. Shin, cond-mat/9912399 (unpublished).

${ }^{26}$ Aharonov-Bohm conductance-oscillations in open rings with in-line quantum dots have found considerable attention following A. Yacoby, M. Heiblum, D. Mahalu, and H. Shtrikman, Phys. Rev. Lett. 74, 4047 (1995). As an entry to the more recent literature we refer the reader to T. Taniguchi and M. Büttiker, Phys. Rev. B 60, 13814 (1999); G.Hackenbroich, Physics Report, (unpublished). cond-mat/0006361

27 R. Deblock, Y. Noat, H. Bouchiat, B. Reulet, and D. Mailly, Phys. Rev. Lett. 84, 5379 (2000).

${ }^{28}$ Weak coupling gives rise to a small coupling coefficient in the Coulomb energy if it is expressed in terms of potentials. If the Coulomb energy is written in terms of the polarization charges weak coupling corresponds to a divergent coupling term in the Coulomb energy.

${ }^{29}$ L. Tian, L. S. Levitov, C. H. van der Wal, J. E. Mooij, T. P. Orlando, S. Lloyd, C. J. P. M. Harmans, and J. J. Mazo, cond-mat/9910062 (unpublished).

30 Ya. M. Blanter and M. Büttiker, Physics Reports, (unpublished), cond-mat/9910158. 